\title{
Pautas y problemas derivados del consumo de fármacos entre los consumidores de éxtasis en Australia
}

\section{Patterns and harms of pharmaceutical drug use among ecstasy users in Australia}

\author{
| Edmund Silins ${ }^{1}$; Jan Copeland²; Paul Dillon²
}

\author{
${ }^{1}$ Centro Nacional para la Investigación sobre el Alcohol y las \\ Drogas; Universidad de Nueva Gales del Sur, Sídney, Australia. \\ ${ }^{2}$ Centro Nacional de Prevención e Información sobre el Cannabis; \\ Universidad de Nueva Gales del Sur, Sídney, Australia.
Enviar correspondencia a:
Edmund Silins. Centro Nacional para la Investigación sobre el Alcohol y las Drogas
Universidad de Nueva Gales del Sur. Sídney, NSW, 2052 Australia.
Tel: 6129385 0141Fax: 61293850222.
E-mail: e.silins@unsw.edu.au

\section{RESUMEN}

Antecedentes: en el ambiente de las discotecas de todo el mundo existe un aumento significativo del uso de medicamentos con receta. El consumo conjunto de éxtasis y fármacos se asocia con daños potencialmente graves para la salud. Esto tiene ramificaciones en el caso de personas que combinan de forma deliberada los fármacos con el éxtasis para lograr un determinado efecto y en quienes toman legalmente fármacos por problemas de salud y de vez en cuando consumen éxtasis. Este estudio exploratorio se propone investigar los hábitos y daños del consumo concomitante de fármacos y éxtasis y explorar las experiencias de los participantes cuando visitan al médico de cabecera (MC). Método: se entrevistó a fondo a 30 consumidores de éxtasis/fármacos. Resultados: los consumidores admitieron la combinación deliberada de estimulantes (dexanfetamina, metilfenidato [p.e. Ritalín]), antidepresivos y sildenafil (p.e. Viagra ${ }^{\circledR}$ ) con éxtasis por motivos no médicos. Se descubrió una amplia gama de efectos. Aparecieron claras relaciones entre el consumo de fármacos y éxtasis, y se analizaron las distinciones entre ellos en términos de motivaciones para el consumo y consecuencias del mismo. Las actitudes de los MC fueron profesionales y sin prejuicios, y los participantes mostraron escasa resistencia a reconocer el consumo de drogas ilegales. Se limitó el cribado de drogas ilegales. Consecuencias: Los resultados tienen importantes repercusiones para el público de las discotecas en lo referente a estrategias de reducción del daño y en el descubrimiento de una serie de ámbitos para futuras investigaciones.

Palabras clave: éxtasis, fármacos con receta, hábitos de consumo, clientes de discotecas, médicos de cabecera, reducción del daño. recibido: noviembre 2008 aceptadp: febrero 2009

\section{ABSTRACT}

Background: There has been a significant incursion of prescription drugs in to the club scene worldwide. The concomitant use of ecstasy and pharmaceutical drugs is associated with potentially serious healthrelated harms. This has ramifications for people who deliberately combine pharmaceutical drugs with ecstasy to achieve a specific effect and for people legitimately using pharmaceutical drugs for a health condition who may incidentally use ecstasy. This exploratory study had the broad aim to investigate the patterns and harms of concomitant use of pharmaceutical drugs and ecstasy, and to explore participants' experiences when visiting a General Practitioner (GP). Methods: In-depth interviews were conducted with 30 ecstasy/pharmaceutical drug users. Results: Users reported deliberately combining stimulants (dexamphetamine, methylphenidate (e.g. Ritalin $\left.{ }^{\circledR}\right)$, antidepressants and sildenafil (e.g. Viagra ${ }^{*}$ ) for non-medical purposes with ecstasy. A wide range of effects were reported. Several clear relationships emerged between the use of pharmaceutical drugs and ecstasy, and the distinctions between these in terms of motivations for use and consequences are discussed. The attitudes of GPs were professional and non-judgemental and there was little reluctance among participants to disclose illicit drug use. Screening for illicit drug use was limited. Implications: Findings have important implications for clubbers in regard to harm reduction strategies and highlight a number of areas for future research.

Key words: ecstasy, prescription drugs, patterns of use, harm reduction, clubbers, General Practitioners. 


\section{INTRODUCCIÓN}

E "éxtasis" (3,4-metilenedioximetanfetamina; MDMA) es una droga ilegal cuyo consumo está muy entendido entre los jóvenes clientes de las discotecas ${ }^{1,2}$. La investigación demuestra que los consumidores de éxtasis practican el policonsumo de drogas ${ }^{3,7}$, y combinan una serie de sustancias con el éxtasis durante el período de "bajón»", investigación de los hábitos del policonsumo de drogas entre los clientes de discotecas se ha centrado fundamentalmente en las drogas ilegales. Pocos estudios han considerado el consumo de fármacos entre este grupo de población ${ }^{8,10,11}$. Se trata de un campo importante, ya que en los últimos años, el uso médico de los psicotrópicos (p.e., antidepresivos, estimulantes, benzodiazepinas) ha aumentado considerablemente en Estados Unidos ${ }^{12}$, Europa ${ }^{13}$, Gran Bretaña ${ }^{14}$ y Australia ${ }^{15}$. La creciente oferta se relaciona con el problema, cada vez mayor, del uso incorrecto de los fármacos ${ }^{16}$, tendencia que se observa en todo el mundo ${ }^{16-19}$.

Los resultados recientes muestran que se ha producido una significativa incursión de los fármacos que requieren receta en el ambiente de las discotecas 7,8,20,21. En Estados Unidos la tasa de los clientes jóvenes de las discotecas que hacen un uso incorrecto de los fármacos con receta es muy alta $^{20}$. Una muestra de 186 consumidores de éxtasis escogidos entre los clientes de discotecas de Miami reveló que más del $80 \%$ habian utilizado de forma incorrecta opioides, sedantes o estimulantes con receta, y el $29 \%$ habian combinado el éxtasis con uno o más fármacos con receta ${ }^{8}$. Entre los clientes de discotecas británicos que consumen drogas recreativas está cada vez más extendida la experimentación con sildenafil (p.e. Viagra $\left.{ }^{\circledR}\right)^{10}$. Más preocupantes son los resultados de un estudio realizado entre 216 consumidores de éxtasis en Australia, en el que se demostró que la cuarta parte habian combinado deliberadamente fármacos junto con éxtasis o los habian tomado después para paliar ciertos efectos secundarios o reforzar el efecto de la droga ${ }^{22}$. Entre 661 consumidores de éxtasis, clientes de discotecas o festivales de música, se observó una notable prevalencia ${ }^{23}$ de la combinación deliberada de antidepresivos (8\%) o estimulantes farmacéuticos (10\%) con el éxtasis para conseguir determinados efectos.

Esta práctica se asocia con daños potencialmente graves para la salud ${ }^{22}$. En concreto, el consumo de ciertos antidepresivos (p.e., IMAO o RIMA) con éxtasis incrementa la posibilidad de padecer sindrome serotoninérgico ${ }^{24}$, una enfermedad potencialmente mortal. En menor medida, el consumo concomitante de estimulantes farmacéuticos con éxtasis también ha provocado sindrome serotoninérgico ${ }^{25}$. Esto tiene consecuencias en las personas que deliberadamente combinan los fármacos con el éxtasis y en las que consumen fármacos legítimamente por cuestiones de salud y que de vez en cuando consumen éxtasis. Por otro lado, investigaciones recientes resaltan la tendencia a asociar el consumo de sildenafil, drogas de discoteca, y conductas de riesgo sexual que derivan en enfermedades de transmisión sexual ${ }^{26,27}$. Estos hábitos tan dañinos de ingestión de fárma-

\section{INTRODUCTION}

- cstasy' (3,4-methylenedioxymethamphetamine; MDMA)

E is a widely used illicit drug popular among young clubbers ${ }^{1,2}$. Research consistently shows that ecstasy users are extensive polydrug users ${ }^{3-7}$, typically using a range of substances together with ecstasy and during the 'come down' period ${ }^{8,9}$. Research into patterns of polydrug use among clubbers has primarily focused on illicit drugs. Few studies have looked specifically at pharmaceutical drug use among this population (for examples see ${ }^{8,10,11}$ ). This is an important area to address, as in recent years, the medical use of psychotrophic drugs (e.g., antidepressants, stimulants, benzodiazepines) has increased considerably in the US ${ }^{12}$, Europe $^{13}$, the UK ${ }^{14}$ and Australia ${ }^{15}$. Increased availability appears to be associated with the growing problem of pharmaceutical drug misuse ${ }^{16}$, a trend that has been observed worldwide ${ }^{16-19}$

Recent evidence suggests there has been a significant incursion of prescription drugs into the club scene ${ }^{7,8,20,}$ ${ }^{21}$. In the United States, club-going young adults misuse prescription drugs at high rates ${ }^{20}$. A sample of 186 ecstasy users recruited from the Miami club scene revealed that more than $80 \%$ had misused prescription opioids, sedatives or stimulants, and $29 \%$ had used one or more prescription drugs together with ecstasy ${ }^{8}$. Among British night-clubbers who use recreational drugs, experimentation with sildenafil (e.g., Viagra ${ }^{\circledR}$ ) appears to have become increasingly established ${ }^{10}$. Of further concern are findings from a survey of 216 ecstasy users in Australia which found a quarter had deliberately used pharmaceutical drugs before, during or after ecstasy use in order to negate certain side-effects or enhance the drug experience ${ }^{22}$. Among 661 ecstasy users attending dance and music festival events, the lifetime prevalence of deliberately combining antidepressants (8\%) or pharmaceutical stimulants (10\%) with ecstasy to achieve a specific effect was notable ${ }^{23}$.

This practice is associated with potentially serious health-related harms ${ }^{22}$. In particular, the use of some antidepressants (e.g., MAOIs and RIMAs) with ecstasy increases the likelihood of serotonin syndrome ${ }^{24}$, a potentially fatal condition. To a lesser degree, concomitant use of pharmaceutical stimulants with ecstasy has also been implicated in serotonin syndrome ${ }^{25}$. This has ramifications for people who deliberately combine pharmaceutical drugs with ecstasy and for people legitimately using pharmaceutical drugs for a health condition who may incidentally use ecstasy. Additionally, recent research is suggestive of an association between sildenafil use, club drugs, sexual risk taking and sexually transmitted infections ${ }^{26,27}$. Such potentially harmful patterns of pharmaceutical drug use among ecstasy users warrant further investigation.

Earlier work among ecstasy users indicates that general medical practitioners (GP) were mainly used for information 
cos por parte de los consumidores de éxtasis merecen investigaciones más profundas.

Trabajos anteriores sobre los consumidores de éxtasis muestran que recurrieron a los médicos de cabecera (MC) fundamentalmente para informarse de los efectos secundarios y posibles daños de drogas como el éxtasis ${ }^{22}$. En general, los MC están en condiciones de reaccionar ante el consumo problemático de drogas y alcohol debido a su accesibilidad, credibilidad y al alto número de pacientes con problemas de consumo de sustancias ${ }^{28,30}$. Sin embargo, hay pocos datos sobre la relación de los MC con los pacientes consumidores de éxtasis. Los consumidores habituales de éxtasis se diferencian mucho de otros consumidores de drogas (p.e., los consumidores de drogas inyectables) en cuanto a edad, educación y pautas de consumo de drogas ${ }^{9,31}$, y por tanto sus necesidades también difieren de las que tienen los consumidores problemáticos de otras drogas. Aparte de eso, existen evidencias del escaso papel de los MC en la detección de las drogas ilegales ${ }^{32-34}$, y por tanto muchos problemas no son detectados o se diagnostican de forma errónea.

Este estudio exploratorio se propone el ambicioso objetivo de investigar los hábitos y daños del consumo concomitante de fármacos y éxtasis (p.e., el consumo de fármacos antes, durante o después de la ingestión de éxtasis). En concreto, pretende investigar el consumo concomitante y deliberado de antidepresivos y éxtasis; y entre los que consumen antidepresivos por enfermedad (p.e. depresión), cómo cambian los hábitos al combinarlos con éxtasis. Se han estudiado también experiencias de consultas con los MC. El estudio obtuvo la aprobación del Comité de Ética sobre Investigación Humana de la Universidad de Nuevas Gales del Sur.

\section{MÉTODO}

Se entrevistó a fondo a 30 consumidores de éxtasis que en fecha reciente habian combinado el éxtasis con los fármacos. La selección se realizó a través de anuncios en publicaciones gratuitas (como revistas de discotecas), páginas web $y$, en menor medida, a través del muestreo en "bola de nieve». Las preguntas del cribado garantizaron que los participantes habían consumido antidepresivos por motivos de enfermedad y éxtasis al menos tres veces en los seis meses anteriores; 0 habian consumido de forma intencionada antidepresivos con éxtasis para lograr un efecto concreto al menos tres veces en los seis meses anteriores; tenían más de 16 años y habian visitado al MC recientemente. Se solicitó el consentimiento informado. Las entrevistas fueron confidenciales y duraron entre 60 y 90 minutos. La participación fue voluntaria, y los participantes recibieron aprox. $18 €$ para gastos de desplazamiento y similares.

La entrevista se centró en la experiencia de los participantes de combinar éxtasis con fármacos y en los daños asociados. Los fármacos analizados fueron: antidepresivos, dexanfetamina/metilfenidato (p.e. Ritalín ${ }^{\circledR}$ ), benzodiazepinas/somniferos y sildenafil). Se investigó la procedencia de los fármacos. Asimismo, se preguntó a los participantes por relating to the side effects and potential harms of drugs such as ecstasy 22 . Generally, GPs are well positioned to respond to problematic drug and alcohol use because of their accessibility, credibility and likely frequent exposure to patients with substance use problems ${ }^{28-30}$. However, little is known about how GPs respond to patients with ecstasyrelated issues. Regular ecstasy users differ markedly from other drug users (e.g., injecting drug users) in relation to age, education and patterns of drug use ${ }^{9,31}$, and therefore, their needs are likely to be different from those of problematic users of other drugs. Additionally, there is evidence that the involvement of GPs in screening for illicit drug use is limited ${ }^{32-34}$, and consequently many problems may remain undetected or be misdiagnosed.

This exploratory study had the broad aim to investigate the patterns and harms of concomitant use of pharmaceutical drugs and ecstasy (e.g., the use of pharmaceutical drugs before, during or immediately after ecstasy intoxication). Specifically, it aimed to investigate the deliberate concomitant use of antidepressants and ecstasy; and, among those using antidepressants for a current health condition (e.g., depression), how patterns of use may change when using ecstasy. Additionally, experiences when visiting a GP were explored. The study had approval of the University of New South Wales Human Research Ethics Committee.

\section{METHODS}

In-depth interviews were conducted with 30 ecstasy users who had recently combined ecstasy and pharmaceutical drugs. Recruitment occurred through advertisements in street press (including clubbing magazines), internet websites, and to a lesser extent, 'snowball' sampling. Screening questions ensured participants: had been using antidepressants for a current medical condition and had used ecstasy on at least three occasions in the last six months; or, had intentionally used antidepressants with ecstasy to achieve a specific effect on at least three occasions in the last six months; and, were at least 16 years of age and had recently visited a GP. Informed consent was obtained. Interviews were confidential and ranged from 60 to 90 minutes. Participation was voluntary and subjects were reimbursed $18 €$ for travel and related expenses.

The interview explored participants' experience of combining ecstasy with pharmaceutical drugs and any associated harms. The pharmaceutical drugs asked about were: antidepressants, dexamphetamine/methylphenidate (e.g., Ritalin ${ }^{\circledR}$ ), benzodiazepines/sleeping tablets and sildenafil. Sources of pharmaceutical drugs were investigated. Questions 
las visitas al MC en las que se les recetaron medicamentos. Finalmente, se explicaron los daños potenciales de consumir éxtasis y fármacos y se ofreció a los participantes la posibilidad de obtener información adicional en caso necesario.

\section{RESULTADOS}

Se alcanzó la saturación de temas con una muestra de 30 participantes.

\section{Demografía}

La mayoría de los participantes tenían poco más de treinta años (edad media, 34; SD 9.8; rango 18-59). La mayoría eran hombres (67\%), nacidos en Australia (77\%) y residentes en zonas urbanas deprimidas (70\%). Dos tercios (67\%) habian cursado bachillerato y un tercio (33\%) tenía una licenciatura universitaria o equivalente.

\section{Enfermedades habituales}

Las enfermedades más frecuentes eran depresión (63\%), ansiedad (20\%) y trastorno bipolar (7\%). Dos participantes (7\%) admitieron el consumo de metilfenidato con fines médicos. La mayoría (87\%) consumía habitualmente antidepresivos por enfermedad. Entre ellos, a la mayor parte $(58 \%)$ les recetaron ISRS (a saber, citalopram, fluoxetina, escitalopram, sertralina) y a los restantes les recetaron "otros" antidepresivos (como mirtazapina 0 rowexetina) (23\%), el ISRN venlafaxina (12\%) y tricíclicos (como amitriptilina, prothiaden)(7\%). No se detectó consumo con fines médicos de IMAO (p.e. moclobemida) or RIMA (p.e. fenelzine, tranilcipromina). Entre los consumidores de antidepresivos por enfermedad, la edad media del diagnóstico fue de 29 años (SD 8,7; rango 14-46).

Cuatro (13\%) participantes superaban 31 puntos en el Inventario de Depresión de Beck (BDI) ${ }^{35,36}$, lo cual indica un elevado nivel de síntomas depresivos. La mayoría (70\%) tenían menos de 17 puntos, lo cual indica bajos niveles de sintomas depresivos. El resto (17\%) tenían entre 17 y 30 puntos, lo cual indica niveles moderados de síntomas depresivos. La media del BDI fue de 13.5 (SD 11.6; rango 0-45).

\section{Hábitos de consumo de drogas ilegales y fármacos}

Se ha observado (Tabla 1) el consumo de una amplia gama de drogas ilegales. En el contexto del consumo deliberado con fines no médicos, un tercio (33\%) de los participantes combinaron antidepresivos con éxtasis, y el $13 \%$ combinaron dexanfetamina o metilfenidato con éxtasis. Es habitual (77\%) el consumo permanente de benzodiazepinas/ somniferos. Aproximadamente dos tercios (37\%) habian consumido sildenafil u otras drogas similares. En consonancia con los criterios de selección, todos los participantes consumían de forma permanente antidepresivos. also related to participants' experiences when they visited a GP and were prescribed pharmaceuticals. On completion, the potential harms of using ecstasy with pharmaceutical drugs were explained and participants were informed about where to access additional information if needed.

\section{RESULTS}

Saturation of themes was achieved with a sample of 30 participants.

\section{Demographics}

Participants were generally aged in their early thirties (mean age 34; SD 9.8; range 18-59). Most were male (67\%), Australian born (77\%) and lived in an innercity location (70\%). Two-thirds (67\%) had completed year 12 and one-third (33\%) held a university or college qualification.

\section{Current health conditions}

The most frequently reported health conditions were depression (63\%), anxiety (20\%) and bipolar disorder (7\%). The medical use of methylphenidate was reported by two (7\%) participants. The majority (87\%) regularly used antidepressant drugs for a current health condition. Of these, most (58\%) were prescribed SSRIs (i.e. citalopram, fluoexitine, escitalopram, sertraline) and the remainder were prescribed 'other' antidepressants (i.e. mirtazapine, robexetine) $(23 \%)$, the SNRI venlafaxine (12\%) and TCAs (i.e. amitriptyline, prothiaden)(7\%). The medical use of MAOIs (e.g. moclobemide) or RIMAs (e.g. phenelezine, tranylcypromine) was not reported. Among those who used antidepressants for a medical condition, the mean age of diagnosis was 29 years (SD 8.7, range 14-46).

Four (13\%) participants had Beck Depression Inventory $(B D I)^{35,36}$ scores of 31 or higher indicating high levels of depressive symptoms. The majority (70\%) had scores below 17 indicating low levels of depressive symptoms. The remainder (17\%) had scores between 17 and 30 suggestive of moderate levels of depressive symptoms. The mean BDI score was 13.5 (SD 11.6; range 0-45).

\section{Patterns of illicit and pharmaceutical drug use}

The use of a wide range of illicit drugs was reported (Table 1). In the context of deliberate use for non-medical purposes, one-third (33\%) of participants had ever used antidepressants with ecstasy, and $13 \%$ had combined dexamphetamine or methylphenidate with ecstasy. Lifetime benzodiazepine / sleeping tablet use was common (77\%). Just over two-thirds $(37 \%)$ had used sildenafil or other similar drugs. Consistent with selection criteria, all participants reported lifetime use of antidepressant drugs. 
Tabla 1: Hábitos de consumo de drogas ilegales y fármacos

Table 1: Patterns of illicit and pharmaceutical drug use

\begin{tabular}{|c|c|c|c|c|c|}
\hline $\begin{array}{l}\text { Droga consumida / } \\
\text { Drug used }\end{array}$ & $\begin{array}{l}\text { Alg. vez N (\%) } \\
\text { Everused N }(\%)\end{array}$ & $\begin{array}{l}\text { Últimos } 5 \text { meses N }(\%) \\
\text { Used in last } 6 \text { months } N(\%)\end{array}$ & $\begin{array}{c}\text { Promedio de dias en los } \\
\text { últimos } 6 \text { meses (rango) } \\
\text { Median days used in last } 6 \text { months } \\
\text { (range) }\end{array}$ & $\begin{array}{l}\text { Cantidad consumo en un episodio } \\
\text { "tipico" } \\
\text { Amount used in a 'typical' episode }\end{array}$ & $\begin{array}{l}\text { Edad media del primer consumo } \\
\text { (SD; rango) } \\
\text { Mean age of first use (SD; range) }\end{array}$ \\
\hline Éxtasis / Ecstasy & $\begin{array}{l}30 \\
(100)\end{array}$ & $\begin{array}{c}30 \\
(100)\end{array}$ & $\begin{array}{c}8 \\
(3-72)\end{array}$ & $\begin{array}{l}\text { 1-4 pastillas } \\
1-4 \text { pills }\end{array}$ & $\begin{array}{c}26 \\
(8.9 ; 15-54)\end{array}$ \\
\hline $\begin{array}{l}\text { Metanfetamina en polvo (speed) / } \\
\text { Methamphetamine powder (speed) }\end{array}$ & $25(83)$ & $\begin{array}{c}9 \\
(30)\end{array}$ & $\begin{array}{c}2 \\
(1-4)\end{array}$ & $\begin{array}{l}1 \text { pinchazo- } 2 \text { gramos } \\
1 \text { point-2 grams }\end{array}$ & $\begin{array}{c}23 \\
(9.0 ; 13-55)\end{array}$ \\
\hline $\begin{array}{l}\text { Metanfetamina cristalina (Ice) / } \\
\text { Crystal methamphetamine (ice) }\end{array}$ & $\begin{array}{l}17 \\
(57)\end{array}$ & $\begin{array}{c}16 \\
(53)\end{array}$ & $\begin{array}{c}6 \\
(1-84)\end{array}$ & $\begin{array}{l}1 \text { pinchazo-20 pipas } \\
1 \text { point-20 pipes }\end{array}$ & $\begin{array}{c}33 \\
(8.8 ; 18-56)\end{array}$ \\
\hline Cocaina / Cocaine & $\begin{array}{c}19 \\
(63)\end{array}$ & $\begin{array}{c}9 \\
(30)\end{array}$ & $\begin{array}{c}2 \\
(1-24)\end{array}$ & $\begin{array}{l}1 \text { raya-2 gramos } \\
1 \text { line-2 grams }\end{array}$ & $\begin{array}{c}25 \\
(9.1 ; 13-55)\end{array}$ \\
\hline GHB / GHB & $\begin{array}{c}12 \\
(40)\end{array}$ & $\begin{array}{c}11 \\
(37)\end{array}$ & $\begin{array}{c}2 \\
(1-72)\end{array}$ & $\begin{array}{l}1-2 \text { ampollas } \\
1-2 \text { vials }\end{array}$ & $\begin{array}{c}33 \\
(6.4 ; 21-43)\end{array}$ \\
\hline Ketamina / Ketamine & $\begin{array}{l}15 \\
(50)\end{array}$ & $\begin{array}{c}12 \\
(40)\end{array}$ & $\begin{array}{c}2 \\
(1-72)\end{array}$ & $\begin{array}{l}\text { 1-5 inhalacion } \\
1-5 \text { bumps }\end{array}$ & $\begin{array}{c}31 \\
(9.2 ; 19-54)\end{array}$ \\
\hline Cannabis / Cannabis & $\begin{array}{l}30 \\
(100)\end{array}$ & $\begin{array}{l}19 \\
(63)\end{array}$ & $\begin{array}{c}24 \\
(1-168)\end{array}$ & $\begin{array}{l}2 \text { conos- } 10 \text { porros } \\
2 \text { cones- } 10 \text { joints }\end{array}$ & $\begin{array}{c}18 \\
(4.6 ; 11-30)\end{array}$ \\
\hline LSD / LSD & $\begin{array}{l}21 \\
(72)\end{array}$ & $\begin{array}{c}3 \\
(10)\end{array}$ & $\begin{array}{c}5 \\
(1-24)\end{array}$ & $\begin{array}{l}0.5-3 \text { pastillas } \\
0.5-3 \text { tabs }\end{array}$ & - \\
\hline Heroina / Heroin & $\begin{array}{c}5 \\
(17)\end{array}$ & $\begin{array}{l}2 \\
(7)\end{array}$ & $\begin{array}{c}4 \\
(2-6)\end{array}$ & $\begin{array}{l}1-2 \text { cigarrillos } \\
1-2 \text { hits }\end{array}$ & $\begin{array}{c}19 \\
(2.7 ; 16-23)\end{array}$ \\
\hline $\begin{array}{l}\text { Antidepresivost / } \\
\text { Antidepressant drugst }\end{array}$ & $\begin{array}{c}10 \\
(33)\end{array}$ & $\begin{array}{c}6 \\
(20)\end{array}$ & $\begin{array}{c}7 \\
(3-14)\end{array}$ & $\begin{array}{l}1-3 \text { pastillas } \\
1-3 \text { tablets }\end{array}$ & $\begin{array}{c}27 \\
(12.9 ; 15-57)\end{array}$ \\
\hline $\begin{array}{l}\text { Dexanfetamina/ metilfenidato }+ \text { I } \\
\text { Dexamphetamine/methylphenidate }+\end{array}$ & $\begin{array}{c}4 \\
(13)\end{array}$ & $\begin{array}{c}2 \\
(7)\end{array}$ & $\begin{array}{c}1.5 \\
(1-2)\end{array}$ & $\begin{array}{l}1-3 \text { pastillas } \\
1-3 \text { tablets }\end{array}$ & $\begin{array}{c}23 \\
(13.7 ; 13-43)\end{array}$ \\
\hline $\begin{array}{l}\text { Benzodiazepinas y somniferous / } \\
\text { Benzodiazepines and sleeping tablets }\end{array}$ & $\begin{array}{l}23 \\
(77)\end{array}$ & $\begin{array}{l}15 \\
(50)\end{array}$ & $\begin{array}{c}6 \\
(1-84)\end{array}$ & $\begin{array}{l}1-5 \text { pastillas } \\
1-5 \text { tablets }\end{array}$ & $\begin{array}{c}27 \\
(8.1 ; 16-43)\end{array}$ \\
\hline $\begin{array}{l}\text { Sildenafil y drogas similares / } \\
\text { Sildenafil and other similar drugs }\end{array}$ & $\begin{array}{c}11 \\
(37)\end{array}$ & $\begin{array}{c}7 \\
(23)\end{array}$ & $\begin{array}{c}3 \\
(1-27)\end{array}$ & $\begin{array}{l}0.3-2 \text { pastillas } \\
0.3-2 \text { tablets }\end{array}$ & $\begin{array}{c}35 \\
(8.1 ; 21-54)\end{array}$ \\
\hline
\end{tabular}

tEn el contexto de un consumo deliberado con fines no medicinales. / In the context of deliberate use for non-medical purposes

\section{Antidepresivos y éxtasis}

Con respecto al consumo concomitante de éxtasis y antidepresivos, se preguntó a los participantes si eran conscientes de los problemas de salud a los que se exponían, si alteraban las horas o los dias en que tomaban los antidepresivos que se les habían recetado cuando planeaban consumir éxtasis, y si habian consumido éxtasis en fechas recientes. La mitad (50\%) admitió conocer los problemas de salud, y las respuestas más habituales fueron:

Mezclar ciertos antidepresivos que actúan sobre la serotonina con éxtasis puede ser peligroso.

Se puede provocar un síndrome serotoninérgico al combinar éxtasis con SSRI o anfetaminas.

No es recomendable, pero no sé por qué. No se deben tomar antidepresivos y drogas al mismo tiempo.

De los 26 participantes (87\%) que declararon que consumian habitualmente antidepresivos por enfermedad, la mayoría (58\%) alteró los horarios o los dias en que debían tomar los antidepresivos porque planeaban consumir éxtasis. Por lo general, los participantes dejaron de tomar antidepresivos unos días antes de consumir éxtasis y volvieron a tomarlos unos dias después. Entre los motivos, adujeron:

Asíno resulta tan peligroso.

Potenciar al máximo el efecto del éxtasis.

\section{Antidepressant drugs and ecstasy}

Regarding the concomitant use of ecstasy and antidepressants, participants were asked about their awareness of associated health problems; and, what changes they made to the times or days they take their prescribed antidepressants if they planned on using ecstasy, or had recently used ecstasy. Half (50\%) mentioned they were aware of health problems and typical responses included:

Mixing some antidepressants that act on serotonin with ecstasy can be dangerous.

You can get serotonin syndrome from using ecstasy with SSRIs or amphetamine.

It's not recommended, but don't know why. You shouldn't take antidepressants with any drugs.

Of the $26(87 \%)$ participants who reported regular use of antidepressant drugs for a medical condition, the majority (58\%) made changes to the times or days they had taken their antidepressant drugs because they planned to use ecstasy. Typically, participants stopped taking antidepressants a few days before using ecstasy and recommenced a few days later. Reasons for this included:

It wouldn't be so dangerous.

To maximise the effect of the ' $e$ '. 


\section{No quería tomar drogas con drogas.}

Estos participantes afirmaron que, si no alterasen la toma de antidepresivos, el "subidón» del éxtasis se reduciria mucho o "prácticamente no habría subidón».

Los restantes (42\%) no alteraron los horarios ni los días en que tomaban antidepresivos cuando consumieron éxtasis. De ellos, la mayoría (82\%) no experimentó ningún efecto negativo. Una minoría (18\%) reconoció haber sufrido efectos negativos. Estos participantes consumieron SSRI y las experiencias negativas fueron:

\section{Las pastillas no surtieron el mismo efecto.}

El bajón fue el doble de malo.

Diez (33\%) participantes tomaron deliberadamente antidepresivos antes, durante o después de consumir éxtasis. Los antidepresivos consumidos en estos casos fueron SSRI, SNRI y el RIMA moclobemida. Se adujeron las siguientes razones:

\section{Alivia el bajón.}

Me convencieron [un amigo] de que potenciaba la serotonina y evitaba la depresión después del éxtasis.

Mi camello dijo que tomar antidepresivos con la última pastilla de la noche hacía que surtiese más efecto y durase más.

La mayoría de los que consumieron deliberadamente antidepresivos con éxtasis lo hicieron durante el «bajón». En general, aumentaron la dosis de antidepresivos prescritos la mañana después de consumir éxtasis. Lo hicieron para contrarrestar la depresión posterior al éxtasis. Dos participantes admitieron el peligrosísimo consumo del RIMA moclobemida con éxtasis. Al parecer, lo hicieron para acrecentar el efecto de las pastillas de éxtasis: retrasaron el efecto del éxtasis, pero el "subidón" fue más fuerte y duró más.

\section{Otros fármacos y éxtasis}

Once (37\%) participantes combinaron sildenafil con éxtasis. Lo hicieron fundamentalmente para prolongar la erección mientras mantenian relaciones sexuales durante o inmediatamente después de una intoxicación aguda de éxtasis. Un participante se quejó de "dolor» debido a la duración de la erección (priapismo). Una mujer consumió sildenafil en un trance similar y declaró que "todo era mucho más intenso".

Unos cuantos participantes (13\%) combinaron estimulantes farmacéuticos como el metilfenidato y la dexanfetamina con el éxtasis. De ese modo adquirieron más energía (p.e. "estás más despierto y piensas con más claridad»), y uno declaró que lo había hecho para "contrarrestar una pastilla de "smacky" [heroina adulterada]". Tras combinar metilfenidato con éxtasis, un participante declaró que "a veces se le revolvía el estómagon. Dos participantes combinaron la dexanfetamina con el éxtasis por motivos similares al metilfenidato.

La gran mayoría de participantes (77\%) consumieron benzodiazepinas o somniferos con éxtasis. De ellos, tres consumieron las benzodiazepinas antes o durante la intoxicación de éxtasis para "colocarse" o para reducir el nerviosismo provocado por el consumo de éxtasis. Dieciséis participantes

\section{Didn't want to be taking drugs on drugs.}

These participants all commented that if they did not make these changes the ecstasy 'high' was significantly reduced or there was 'almost no high'.

The remainder (42\%) did not make changes to the times or days they took their antidepressant drugs when using ecstasy. Of these, most ( $82 \%)$ never experienced any negative effects. A minority (18\%) did report negative effects. These participants were using SSRIs and the negative experiences were:

The pills didn't work as well.

The comedown was twice as bad.

Ten (33\%) participants had deliberately taken antidepressant drugs before, during or after ecstasy use. The antidepressants used in this way were SSRIs, SNRIs and the RIMA moclobemide. Typical reasons for this use included:

It helps with the comedown.

Was led to believe [by a friend] it was a serotonin booster to avoid depression after ' $\mathrm{e}$ '.

My dealer said that if you take antidepressants with the last pill of the night it works better and lasts longer.

Most participants who deliberately used antidepressants with ecstasy did so whilst 'coming down'. Typically, they increased their prescribed antidepressant dose in the morning after using ecstasy. This was to counteract postecstasy depression. The extremely risky practice of using the RIMA moclobemide with ecstasy was reported by two participants. Supposedly, this was to increase the effect of multiple ecstasy pills - delaying the onset of ecstasy but resulting in a bigger 'high' which lasted longer.

\section{Other pharmaceutical drugs and ecstasy}

Eleven (37\%) participants had combined sildenafil with ecstasy. This was primarily to maintain an erection while engaging in sexual activity during or immediately after acute ecstasy intoxication. One participant reported 'soreness' from being erect for an extended period of time (priapism). One female had used sildenafil in this way and reported that 'it kept things going a lot longer'.

Few (13\%) participants had combined pharmaceutical stimulants such as methylphenidate and dexamphetamine with ecstasy. Typically, this provided participants with more energy (e.g., 'to make you more alert and think clearly') and one stated it was to "counteract a 'smacky' [heroin adulterated] pill". After combining methylphenidate with ecstasy, one participant reported 'it can sometimes make me sick in the stomach'. Dexamphetamine was used with ecstasy by two participants for similar reasons as methylphenidate.

The large majority (77\%) of participants had used benzodiazepines or sleeping tablets with ecstasy. Of these, three had used benzodiazepines before or during ecstasy intoxication to 'bomb you out' or to reduce the anxiety associated with ecstasy use. Sixteen participants had taken 
tomaron benzodiazepinas o somníferos después de consumir éxtasis para inducir el sueño.

\section{Procedencia de los fármacos}

La mayoría de los participantes (70\%) obtuvieron los fármacos sin receta en algún momento. Entre ellos, los "amigos» fueron la principal fuente de suministro (60\%), seis participantes (20\%) compraron los fármacos a un traficante y uno $(3 \%)$ los adquirió a través de Internet. Otros declararon que, para tratar su enfermedad, consumian habitualmente fármacos recetados a sus parejas. Los fármacos más habituales fueron las benzodiazepinas. Otros medicamentos obtenidos sin receta fueron el sildenafil, una serie de antidepresivos y somniferos.

\section{Experiencias en la consulta del médico de cabecera}

A los participantes se les preguntó por sus experiencias en la consulta del MC. La mayoría (67\%) habian informado a su MC de su consumo de éxtasis y dieron las siguientes razones para hacerlo:

Hace mucho que conozco a mi MC.

Estaba deprimido, y el médico me preguntó si consumía drogas.

Mi médico me vio [en la discoteca] y estuvimos hablando [en el local].

Le pedí que me ayudase a dejar las drogas.

Diecisiete participantes afirmaron que el MC había respondido de forma muy profesional y sin prejuicios. Tres declararon que el MC había reaccionado negativamente, por ejemplo:

Sacudió la cabeza, preguntó cuántas veces consumía y se mostró incómodo al hablar del tema.

Me miró con una cara "decepcionante».

Diez participantes no comunicaron a su MC que consumían éxtasis, y las razones aducidas fueron:

No quiero que me trate como un drogadicto.

En ese momento no consumía drogas.

No hacía falta, puesto que no tengo problemas con el éxtasis.

El médico no me lo preguntó.

Dos tercios de los participantes (67\%) no dudarian en pedir a su MC información sobre los efectos y daños del éxtasis. A un tercio (33\%) les resultaría violento por los siguientes motivos:

EI MC me lo desaconsejaría [el consumo de drogas].

No quiero que el médico pierda el tiempo.

Prefiero mirar en Internet... o hablar con mis amigos o mi camello. benzodiazepines or sleeping tablets after ecstasy use to assist with sleep.

\section{Sources of pharmaceutical drugs}

Most (70\%) participants had obtained pharmaceutical drugs without prescription at some time. Of these, 'friends' were the main source (60\%), six (20\%) participants had acquired pharmaceutical drugs through a drug dealer and one (3\%) had acquired them over the internet. One other reported that, to treat their own health condition, they regularly used pharmaceutical drugs which had been prescribed to their partner. Benzodiazepines were the most frequently attained. Other drugs obtained without prescription were sildenafil, a range of antidepressants and sleeping tablets.

\section{Experiences when visiting a general medical practitioner}

Participants were asked about their experiences when they visited a GP. The majority (67\%) had told their GP about their ecstasy use and typical reasons for how this came about were:

I have a long-standing connection with my GP.

I was depressed and the doctor asked about my drug use.

My doctor's seen me out [clubbing] and we've chatted about it [at the night club].

I asked for help to stop using drugs.

Seventeen participants mentioned that the GP responded in a very professional and non-judgemental way. Three participants reported that the GP responded negatively, for example:

He shook his head, asked how often I used, seemed uncomfortable to discuss it.

He gave me a 'disappointing' look.

Ten participants had not told their GP about their ecstasy use, and typical reasons included:

I didn't want to be treated like a drug addict.

I wasn't using drugs at the time.

There's no need to, I don't have a problem with ecstasy.

He hasn't asked.

If participants had questions about the effects and harms of ecstasy, two-thirds (67\%) would feel comfortable raising them with their GP. The one-third (33\%) who would not feel comfortable, typically reported this was because:

The GP would advise against it [drug use].

I don't want to waste the doctor's time

I'd look on the internet first... or ask my friends or dealer. 


\section{Experiencias con fármacos de prescripción}

La mayoría de los participantes (70\%) habian acudido al MC, que les recetó antidepresivos. Trece de ellos afirmaron que, durante la consulta, el MC no les había preguntado por su consumo de éxtasis, a pesar de que, según ellos, el MC sabía que consumían la droga ilegal. Nueve participantes declararon que el MC les había hecho preguntas sobre su consumo de éxtasis, generalmente relacionadas con la variedad y cantidad de las drogas ilegales y sobre qué pensaban y sentían al consumirlas. Veinte participantes (67\%) afirmaron que la primera vez que acudieron al MC y les recetó antidepresivos, presentaban síntomas de depresión. El MC discutió alternativas a la toma de antidepresivos con seis participantes (20\%), entre ellas la terapia psicológica, la administración de hierba de San Juan y otras terapias naturales, ejercicio, dieta y reducción del consumo de alcohol.

Aproximadamente a la cuarta parte de los participantes $(23 \%)$ que visitaron al MC, se les recetó sildenafil o fármacos similares. El MC no preguntó por el consumo de drogas a ninguno de los cinco participantes a los que se recetó sildenafil; sin embargo, cuatro afirmaron que el MC sabía que consumian drogas. EI MC trató a dos participantes que presentaban síntomas de disfunción eréctil. De los cinco no evaluados, dos afirmaron que el MC sabía que solicitaban sildenafil para paliar los sintomas de la disfunción eréctil que padecian temporalmente debido al consumo de drogas ilegales y que no sufrían dichos sintomas en otras ocasiones. Un participante recurrió al sildenafil para contrarrestar la disfunción eréctil surgida como efecto secundario de los antidepresivos.

La mitad de los participantes (47\%) acudieron a un MC que les recetó benzodiazepinas o somníferos. Aunque la mayoría declaró que el MC no había preguntado si consumían éxtasis en la época de la consulta, varios participantes afirmaron que el MC conocía dicha información. Diez participantes afirmaron que el MC los habia tratado porque presentaban sintomas de ansiedad o insomnio. A seis participantes se les ofrecieron alternativas a las benzodiazepinas 0 a los somníferos, entre ellas dejar de consumir drogas ilegales, terapia psicológica y consumo de suplementos herbales como la valeriana.

\section{DISCUSIÓN}

El estudio reveló hábitos preocupantes de consumo de fármacos y drogas ilegales. La combinación de medicamentos de prescripción con éxtasis fue relativamente habitual, a pesar de conocerse las potenciales repercusiones sobre la salud. Surgieron una serie de claras relaciones entre el consumo de fármacos y el éxtasis. Las distinciones entre ellas en términos de razones para consumir y consecuencias merecen una discusión más profunda. En general, las actitudes de los MC fueron profesionales y sin prejuicios, y hubo poca resistencia a admitir el consumo de drogas ilegales. Prácticamente no se realizó un cribado de drogas ilegales a la hora de recetar fármacos.

\section{Experiences when prescribed pharmaceutical drugs}

Most (70\%) participants had visited a GP and received a prescription for antidepressant drugs. Of these, thirteen reported that during the consultation the GP did not ask about ecstasy use. However, three mentioned that the GP was already aware of their illicit drug use. Nine participants reported that the GP did ask about the use of ecstasy, and questions typically related to the range and quantity of illicit drugs used and the participant's thoughts and feelings on drug use. Twenty (67\%) participants reported that on the first occasion they visited a GP and received a prescription for antidepressant drugs they were assessed for symptoms of depression. The GP discussed alternatives to taking antidepressants with six (20\%) participants, and these typically included counselling, use of St. John's wort and other natural therapies, exercise, diet and reducing alcohol consumption.

About one-quarter (23\%) of participants had visited a GP and received a prescription for sildenafil or other similar drugs. None of the five participants who had recently been prescribed sildenafil reported that the GP had asked about their use of ecstasy, however, four participants commented that the GP already knew of their drug use. Two participants reported that the GP assessed them for symptoms of erectile dysfunction. Of the five who were not assessed, two stated that they had made the GP aware that the request for sildenafil was related to symptoms of erectile dysfunction temporarily experienced during illicit drug use and that symptoms were not present at other times. For one participant, the use of sildenafil was to counteract the erectile dysfunction which can be a side-effect of antidepressant drugs.

Half (47\%) of the participants had visited a GP and received a prescription for benzodiazepines or sleeping tablets. Although a majority reported that the GP did not ask about their use of ecstasy at the time of the consultation, several participants commented that the GP already knew this information. Ten participants reported that the GP did assess them for symptoms of anxiety or sleeplessness. Alternatives to taking the benzodiazepines or sleeping tablets which were prescribed were discussed with six participants, these included cessation of illicit drug use, therapy and use of herbal supplements such as valerian.

\section{DISCUSSION}

The study revealed patterns of illicit and pharmaceutical drug use which are of some concern. Prescription drug use with ecstasy was relatively common despite the awareness of potential health problems. Several clear relationships emerged between the use of pharmaceutical drugs and ecstasy. The distinctions between these in terms of motivations for use and consequences warrant further discussion. Overall, the attitudes of GPs were professional and non-judgemental and there was little reluctance to disclose illicit drug use. Screening for illicit drug use was infrequent when pharmaceuticals were prescribed. 
Las características de la muestra indican que la población del estudio estaba relacionada con el ambiente de discotecas. Los hábitos de consumo de drogas ilegales son similares a los observados en estudios anteriores sobre clientes de discotecas ${ }^{7}$ y consumidores de éxtasis ${ }^{5,37,38}$. Destaca el consumo de cannabis y metanfetamina. El consumo de cocaína es relativamente bajo, característica habitual entre los consumidores australianos de éxtasis ${ }^{3,9}$. La mayoría de los participantes vivian en zonas urbanas deprimidas. La selección se hizo a través de revistas de discotecas, aunque no exclusivamente. Por otro lado, los estudios sobre los consumidores habituales de éxtasis en Australia muestran sin lugar a dudas que las discotecas son los lugares en los que se consume éxtasis con mayor frecuencia ${ }^{9}$. Los participantes del estudio rondaban los treinta años $y$, por tanto, su edad era levemente superior a la de los que participan habitualmente en los estudios sobre consumidores de éxtasis ${ }^{7,39}$. Esto podría ser consecuencia de los criterios de selección, que buscaban participantes que combinasen habitualmente el consumo del éxtasis con los antidepresivos. Dicho hábito, potencialmente peligroso, tiende a ser más común entre personas con más experiencia y, por tanto, entre consumidores de éxtasis de más edad. A la hora de generalizar las conclusiones a clientes de discoteca de otros grupos de edad, ha de tenerse en cuenta que los consumidores más jóvenes y de menor experiencia pueden utilizar estrategias distintas para combinar fármacos con éxtasis.

Existen tres claras relaciones entre el consumo de fármacos y éxtasis. La primera se observó en los que consumían legalmente fármacos con receta por su enfermedad y que alteraron la toma de la medicación para consumir éxtasis. Generalmente, dejaron de tomar antidepresivos unos dias antes de consumir éxtasis y volvieron a tomarlos después. Algunos adoptaron una estrategia de reducción del daño para minimizar los perjuicios asociados al consumo concomitante (p. e., No quería tomar con drogas con drogas), mientras que otros alteraron las pautas para evitar la interacción de drogas que redujese el "subidón" del éxtasis (p.e.,Potenciar el efecto del éxtasis), cosa que suele ocurrir cuando se toman ISRS ${ }^{24,40,41}$. Experiencias previas de combinar éxtasis con antidepresivos influyeron en las prácticas de este grupo. Estos participantes habian experimentado un subidón menor de éxtasis en ocasiones anteriores de consumo concomitante, lo cual significa que este grupo creía que se produciria una interacción si consumian éxtasis y tomaban antidepresivos. Los sujetos no declararon haber sufrido sintomas negativos al dejar los antidepresivos de repente. En parte podría deberse a la vida media, relativamente larga, de algunos ISRS (p. e., fluoxetina: 1-3 dias después de la administración aguda, 4-6 dias después de la administración crónica ${ }^{42}$ ), que atenúa el efecto de las dosis omitidas.

La segunda relación surge entre los que consumieron fármacos prescritos legalmente por una enfermedad y que no dejaron de tomar antidepresivos cuando consumieron éxtasis. Se registraron pocos efectos negativos, lo cual contradice en parte investigaciones anteriores ${ }^{22}$. Generalmente, los efectos negativos consistieron en un "subidón" de éxtasis muy reducido o en un "bajón" más acentuado. La nota-
Sample characteristics indicate that the study population was affiliated with the club scene. Patterns of illicit drug use were similar to those reported in previous studies of clubbers 7 and ecstasy users ${ }^{5,37,38}$. The use of cannabis and methamphetamine was particularly prevalent. Cocaine consumption was relatively low, which is a typical finding among ecstasy users in Australia ${ }^{3,9}$. Most participants were inner-city dwellers. Recruitment occurred through, but was not limited to, clubbing magazines. Additionally, surveys of regular ecstasy users in Australia consistently show that nightclubs are the most common location of ecstasy use ${ }^{9}$. Participants were, however, generally aged in their early30s and, therefore, slightly older than those usually recruited in studies of ecstasy users ${ }^{7}, 39$. This may be a reflection of selection criteria which ensured participants had regularly used ecstasy with antidepressants. This potentially risky practice could be more common among experienced and, therefore, older ecstasy users. When generalising findings to clubbers of other age groups, it must be kept in mind that younger, less experienced users may have different strategies for combining pharmaceutical drugs with ecstasy.

Three clear relationships emerged between the use of pharmaceutical drugs and ecstasy. The first was among those who used legitimately prescribed drugs for a medical condition who then made changes to the use of their medication when they took ecstasy. Typically, these changes included stopping antidepressants in the days leading up to using ecstasy and re-starting again afterwards. While some were adopting a harm reduction strategy to minimise the harms associated with concomitant use (e.g., Didn't want to be taking drugs on drugs), others were making these changes to avoid the drug-drug interaction which may result in a reduced ecstasy 'high' (e.g., To maximise the effect of the 'e'), which is particularly likely if using SSRIs ${ }^{24,40,41}$. Previous experiences of combining ecstasy with antidepressants appeared to influence the current practices of this group. These participants had experienced a diminished ecstasy high on a previous occasion of concomitant use. This implies that this group strongly held the belief that there would be an interaction if they used ecstasy and took antidepressants in the usual way. Participants did not report any negative symptoms from sudden discontinuation of antidepressants. This may partly be accounted for by the relatively long elimination half-life of some SSRIs (e.g., fluoxetine: 1-3 days after acute administration, 4-6 days after chronic administration ${ }^{42}$ ), which may blunt the effect of missed doses.

The second relationship emerged among those who used legitimately prescribed pharmaceuticals for a medical condition who did not make changes to their use of antidepressants when they took ecstasy. Few negative effects were experienced which is somewhat contrary to earlier research ${ }^{22}$. Typically, the negative effects reported were a significantly diminished ecstasy 'high' or a worse 'comedown'. The notable lack of serious negative health 
ble falta de consecuencias negativas graves para la salud no debe sorprender puesto que la mayoría de los participantes tomaban regularmente ISRS o ISRN por motivos de enfermedad, y cuando se combinan con el éxtasis no representan un gran peligro para la salud ${ }^{25}$. A ningún participante se le había prescrito la generación más antigua de antidepresivos (p.e. IMAO o RIMA), que combinados con el éxtasis producen graves aumentos de la serotonina ${ }^{25,41,43}$. Esto concuerda con los informes que indican que estos antidepresivos representan un porcentaje relativamente bajo de las ventas totales de antidepresivos en Australia ${ }^{15}$. La ausencia de experiencias negativas en ocasiones previas de consumo concomitante sin duda influyó en las prácticas habituales de este grupo. Otra posibilidad es que no conociesen las posibles interacciones y, por tanto, no pensasen que su experiencia con el éxtasis podía alterarse. Los resultados del estudio confirman que un condicionante aprendido lleva a los consumidores de éxtasis a decidir si cambian o no su medicación antidepresiva.

Se observó una tercera relación evidente entre los que consumieron fármacos antes, durante o después del éxtasis para evitar ciertos efectos secundarios o potenciar la droga. El consumo deliberado de antidepresivos con fines no médicos para lograr un efecto determinado fue raro. Una minoría aumentó la dosis prescrita de SSRI al día siguiente de consumir éxtasis para contrarrestar el desánimo y el «bajón». En Estados Unidos se han observado prácticas similares entre los consumidores de éxtasis y metanfetamina ${ }^{11}$. No se observó gran incidencia del consumo de RIMA con éxtasis para intensificar y prolongar la intoxicación, práctica potencialmente peligrosa, lo cual coincide con otros estudios sobre este tipo de conducta ${ }^{22}$. Los pocos que combinaron RIMA con éxtasis eran consumidores de más edad y experiencia que conocian los posibles riesgos. Transmitir mensajes de reducción del daño a estos individuos es muy difícil. Curiosamente, varios participantes dijeron que habian combinado ISRS con éxtasis para potenciar el "subidón» del éxtasis. Previamente, en un estudio controlado en el que se utilizaron medidas psicométricas sofisticadas, Letchi et al. (2000) observaron que el ISRS citalopram reducia la intensidad de la intoxicación por MDMA en casi el 60 por ciento de los humanos ${ }^{44}$. Nuestro estudio indica que en las respuestas de los participantes pudieron influir efectos previamente asumidos de las drogas o la no identificación de la droga, lo cual subraya la importancia de una identificación adecuada de las drogas y de la medición objetiva de los efectos de las mismas.

Las ideas sobre los efectos de los antidepresivos combinados con éxtasis iban desde lo razonable (p.e., El consumo de éxtasis con SSRI o anfetaminas puede producir sindrome serotoninérgico) a posturas vagas y poco concretas (p. e., No es recomendable, pero no sé por qué. No se deben tomar antidepresivos con drogas), lo cual indica que hay campo para enseñar los posibles daños a los clientes de las discotecas. La reabsorción de inhibidores de la serotonina (p. e., ISRS, IRSN) influye de forma menos peligrosa en los niveles de serotonina cuando se consume con éxtasis, ya que estos fármacos compiten con el MDMA en el punto receptor de la serotonina y, por tanto, reducen los efectos del éxtasis ${ }^{24,40,41}$. Por el contrario, el consumo de éxtasis con moclobemida (RIMA) e consequences is not surprising given that most participants were regularly using SSRIs or SNRIs for their current health condition, which when used with ecstasy are of relatively low risk to health ${ }^{25}$. No participants had been prescribed older generation antidepressants (e.g. MAOIs or RIMAs), which when used with ecstasy are more likely to result in serious elevations in serotonin ${ }^{25,41,43}$. This is in keeping with reports that these antidepressants represent a relatively small percentage of total antidepressant sales in Australia ${ }^{15}$. It appears that a lack of negative experiences on previous occasions of concomitant use may have influenced current practices among this group. Alternatively, it could be that they were unaware of the potential for interaction and did not hold the belief that their ecstasy experience would be affected. Study findings strongly suggest there is a learned determinant to whether or not ecstasy users made changes to their antidepressant regime.

A third relationship was evident among those who used pharmaceutical drugs before, during or after ecstasy to negate certain side-effects or enhance the drug experience. Deliberately using antidepressant drugs for non-medical purposes to achieve a specific effect was uncommon. A minority increased the prescribed dose of SSRIs the morning after using ecstasy to counteract the depressed mood and 'comedown'. Similar practices among ecstasy and methamphetamine users have been reported in the United States ${ }^{11}$. The use of RIMAs with ecstasy to intensify and prolong intoxication, a potentially risky practice, was not widely reported and in keeping with other studies of this behaviour ${ }^{22}$. The few who combined RIMAs with ecstasy were older, more experienced users who appeared to be aware of the risks involved. Reaching such individuals with harm reduction messages poses unique challenges. Unexpectedly, several participants reported combining SSRIs with ecstasy for the purported effect of potentiating the ecstasy 'high'. Previously, in a controlled study using sophisticated psychometric measures, Letchi et al (2000) found that the SSRI citalopram reduced the intensity of MDMA intoxication by about 60 percent in humans ${ }^{44}$. Our findings suggest that participant responses may have been influenced by learned drug effects or the lack of drug identification. This highlights the importance of accurate drug identification and the objective measurement of drug effects.

Beliefs about the effects of combining antidepressants with ecstasy ranged from relatively valid (e.g., You can get serotonin syndrome from using ecstasy with SSRIs or amphetamine) to non-specific and vague (e.g., It's not recommended, but I don't know why. You shouldn't take antidepressants with any drugs), suggesting there is scope to educate clubbers about the potential harms. Serotonin re-uptake inhibitors (e.g., SSRIs, SNRI) are least likely to increase serotonin to life-threatening levels if used with ecstasy, as these drugs compete with MDMA at the serotonin receptor site and, therefore, diminish the effects of ecstasy $24,40,41$. On the other hand, the use of ecstasy 
IMAO puede producir graves aumentos de la serotonina ${ }^{41,45}$, porque estos antidepresivos combaten la disminución de la serotonina. Consumir éxtasis (liberador de la serotonina) con otros liberadores de la serotonina como las anfetaminas aumenta la probabilidad de sufrir síndrome serotoninérgico ${ }^{25}$. La variedad de resultados del BDI indica que los participantes sufrieron síntomas depresivos de diversa gravedad, lo cual se reflejó en las diferentes dosis de antidepresivos prescritos. La sintomatología depresiva y las dosis de antidepresivos son factores que alteran las reacciones producidas por el consumo de éxtasis, cosa que debe tenerse en cuenta a la hora de interpretar los resultados. En general, el primer consumo de éxtasis (a los 26 años, edad media) precedió al diagnóstico de problemas mentales como depresión (29 años, edad media). Por tanto, cabe la posibilidad de que una proporción del consumo de éxtasis se deba a la automedicación de problemas mentales preexistentes. Asimismo, el consumo de antidepresivos se debe a veces a la reducción de la serotonina producida por el éxtasis. No se recabaron opiniones de los participantes sobre el particular, y es un interesante campo para futuras investigaciones.

Con el éxtasis se consumieron asimismo una serie de fármacos de prescripción. El extendido consumo de benzodiazepinas y somniferos durante el período de "bajón" indica que fue habitual tomar medicación para los efectos residuales y subagudos del éxtasis. Puede ser consecuencia de los hábitos, relativamente contundentes, de consumo de éxtasis entre la muestra, ya que los mayores consumidores de éxtasis consumieron también más fármacos sedantes-relajantes ${ }^{5}$. La automedicación para aliviar los síntomas del «bajón» no es mala idea, pero la evidencia sobre la mejor estrategia para recuperarse de la intoxicación por éxtasis resulta equívoca.

Menos de la cuarta parte habian consumido sildenafil en los seis meses anteriores, y el promedio de días de consumo fue bajo, lo cual no difiere de un estudio sobre clientes de discotecas británicos, entre los cuales el consumo de sildenafil era infrecuente o transitorio $0^{10}$. En el presente estudio, si bien no fueron frecuentes los efectos adversos, un participante sufrió priapismo (dolencia potencialmente grave). Se observaron interacciones entre el sildenafil y otros fármacos de prescripción ${ }^{46,47}$. Resulta preocupante que el MC prescribiese sildenafil como tratamiento para la disfunción eréctil provocada por las drogas, lo cual indica el desconocimiento de las posibles interacciones o que el paciente engañó al MC, no comentando que la disfunción coincidía con períodos de consumo agudo. Un estudio reciente caracterizó el alcance y daños del abuso de sildenafil y destacó que su consumo concomitante con drogas ilegales incrementaba el riesgo de sufrir complicaciones cardiovasculares ${ }^{48}$. Por otro lado, la administración de sildenafil sólo se recomienda en hombres, ya que su administración a mujeres da lugar a riesgos desconocidos. Los investigadores observaron que las asociaciones entre sildenafil, conductas sexuales de riesgo e infecciones de transmisión sexual (véanse ${ }^{26,27}$ ) no se limitaban a hombres que mantenian relaciones con hombres y, por tanto, las consecuencias para la salud pública son más amplias de lo que se pensó en principio ${ }^{10,49}$. Estos aspectos exigen estudios pos- with moclobemide (RIMA) and MAOls may lead to serious increases in serotonin ${ }^{41,45}$, as these antidepressants prevent serotonin breakdown. Using ecstasy (a serotonin releaser) with other serotonin releasers such as amphetamine increase the likelihood of serotonin syndrome ${ }^{25}$. The range of BDI scores suggests participants had depressive symptoms of varying severity, which was reflected in differences in prescribed antidepressant dose. Depressive symptomatology and antidepressant drug dose are factors likely to affect responses to ecstasy use, which must be kept in mind when interpreting results. Generally, first ecstasy use (mean age 26 years) preceded diagnosis of mental health problems such as depression (mean age 29 years). It is plausible, therefore, that a proportion of ecstasy use may have been due to self medication of pre-existing mental health problems. Likewise, antidepressant use may have been due to ecstasy-related serotonin depletion. Respondents' views on this were not sought and this would be an interesting avenue for future research.

A range of other prescription drugs were also used with ecstasy. The widespread use of benzodiazepines and sleeping tablets during the 'comedown' period suggests it was common to medicate the residual, or sub-acute, effects of ecstasy. This may be a reflection of the relatively heavy patterns of ecstasy use among the sample, as heavier ecstasy users report significantly greater use of sedative-relaxant drugs $^{5}$. Whilst self-medicating the symptoms associated with the 'comedown' may seem like a good idea, evidence regarding what might be the best strategy to recover from ecstasy intoxication is equivocal.

While just under one-quarter had used sildenafil in the previous six months, the median days of use were generally low. This was not dissimilar to a survey of British clubbers which found most sildenafil use to be infrequent or transient 10. In the present study, although adverse effects were not common, priapism (a potentially serious condition) was reported by one participant. Interactions between sildenafil and other prescribed drugs have been noted ${ }^{46,47}$. It is concerning that GPs prescribed sildenafil as a treatment for drug induced erectile dysfunction. This suggests a lack of knowledge regarding potential drug interactions, or possibly the GP had been misled by the patient - who may not have disclosed that the dysfunction was related to periods of acute intoxication. A recent review characterised the extent and harms of sildenafil misuse and raised concerns that concomitant use with illicit drugs may increase the risk of cardiovascular complications ${ }^{48}$. Additionally, sildenafil is currently approved only for use in men so use in women would be associated with unknown risks. Commentators have also suggested that associations between sildenafil, risky sexual behaviours and sexually transmitted infections $\left(\right.$ see $^{26,27}$ ) may not be limited to men who have sex with men, and, therefore, the public health implications may be broader than first thought ${ }^{10,49}$. These various aspects require further 
teriores para delinear con mayor claridad las consecuencias del abuso de sildenafil.

Apenas se combinaron estimulantes farmacéuticos con éxtasis y se registraron escasos efectos negativos. Un participante combinó metilfenidato con éxtasis para contrarrestar una pastilla de heroína adulterada. Si bien el metilfenidato se puede utilizar para aliviar la somnolencia y la desorientación -conocidos efectos del éxtasis ${ }^{50,51}$-, es improbable que el adulterante fuese heroína, pues no hay pruebas de que existan pastillas de éxtasis de esa variedad ${ }^{52-54}$. Generalmente los estimulantes farmacéuticos se utilizaron para paliar los efectos menos deseables del éxtasis (p. e., Estar más despierto y pensar con claridad). Investigaciones recientes indican que el consumo de dexanfetamina propicia en ocasiones un mayor policonsumo de drogas al reducir la sensibilidad ante otras drogas (incluyendo el alcohol), lo cual aumenta los daños derivados de dicho consumo (p.e., conducir embriagado, daños agudos asociados a las drogas y al alcohol ${ }^{55}$. El daño potencial de los estimulantes farmacéuticos en el policonsumo de drogas exige una atención concreta y campañas educativas dirigidas a los clientes de discotecasLos amigos y los traficantes de drogas son las principales fuentes de fármacos que se obtienen sin prescripción, como se ha visto en otros estudios sobre la población asistente a las discotecas $^{11}$ y sobre los consumidores de éxtasis ${ }^{22}$. Esto indica la existencia de un activo mercado callejero de fármacos en los ambientes de discoteca, siendo los traficantes de drogas ilegales la principal fuente de suministro. Entre los consumidores de drogas de Miami con edades de 18 a 45 años, los grupos focales manifestaron que los traficantes locales solian "ofrecer" éxtasis con opioides de prescripción o depresores en línea con la demanda de drogas de prescripción para facilitar la recuperación posterior al consumo de éxtasis ${ }^{11}$. Internet no fue un recurso popular para comprar fármacos de prescripción. Aunque su falta de popularidad no se ha analizado en el presente estudio, existen pruebas de que se evita deliberadamente la compra de drogas farmacéuticas en Internet debido a las "estafas» y porque los fármacos de prescripción son más baratos en la calle ${ }^{11}$.

Las conclusiones mayoritariamente positivas sobre el papel de los MC resultaron inesperadas. Las investigaciones sobre los consumidores de drogas ilegales indican que existe bastante resistencia a reconocer el consumo de drogas ilegales ante los profesionales de la medicina debido a dudas sobre la confidencialidad y al temor a recibir un trato peor ${ }^{56-}$ ${ }^{58}$, lo cual se observa en las respuestas de algunos participantes en este estudio. $Y$ en efecto, cuando los consumidores de sustancias acuden al médico, abundan las experiencias de discriminación y de una atención de menor calidad ${ }^{59}$. Varios factores explican que los participantes de este estudio hayan sido más proclives a comunicar el consumo de éxtasis a su MC. Un entorno urbano y no rural, que asegura un considerable anonimato y con mayor población de paso, contribuye a que sea más fácil confesar el consumo de drogas ilegales. Por otro lado, no todos los consumidores de drogas ilegales sufren el mismo estigma. El consumo de éxtasis se considera más "aceptable», lo cual ha contribuido a que se confiese sin problema o a una reacción más positiva del MC. También hay study to more clearly delineate the adverse consequences of sildenafil misuse.

Pharmaceutical stimulants were not commonly used with ecstasy and few negative effects were reported. One participant combined methylphenidate with ecstasy to counteract a 'smacky' [heroin adulterated] pill. While methylphenidate may have been used to counteract drowsiness and disorientation - known effects of ecstasy 50,51 - it is unlikely that the adulterant was heroin as there is little evidence such ecstasy pills exist ${ }^{52-54}$. Typically, pharmaceutical stimulants were used to mask the less desirable effects of ecstasy (e.g., To make you more alert and think clearly). Recent research suggests dexamphetamine use may facilitate greater polydrug use by reducing sensitivity to other drugs (including alcohol), which may increase the harms associated with such use (e.g., driving while intoxicated, acute drug- and alcohol-related harm $)^{55}$. The potentially harmful role of pharmaceutical stimulants in polydrug use is an area which requires specific attention in education campaigns aimed at clubbers.

Friends and drug dealers were prominent sources of pharmaceutical drugs obtained without prescription, as reported in other studies of club-based populations ${ }^{11}$ and ecstasy users ${ }^{22}$. This suggests that an active street market exists for prescription drugs among the club scene with illicit drug dealers a major route of supply. Among 18-45 year old club drug users in Miami, focus groups revealed that local dealers often 'packaged' ecstasy with prescription opioids or depressants in line with demand for prescription drugs to ease post-ecstasy recovery ${ }^{11}$. The Internet was not a popular source of prescription drugs. Although its apparent lack of appeal was not explored in the present study, there is evidence that the Internet is deliberately avoided as a source of pharmaceutical drugs because of 'rip offs' and because prescription drugs were usually cheaper on the street ${ }^{11}$.

The generally positive findings in regard to the role of GPs were somewhat unexpected. Research among illicit drug users tends to indicate there is a reluctance to disclose illicit drug use to medical professionals due to concerns about confidentiality and fear of poor treatment ${ }^{56-58}$. The responses provided by some individuals in the study reflect this. Furthermore, when substance users do seek medical help, experiences of discrimination and lesser quality of care are common ${ }^{59}$. Several factors may account for the majority disclosing ecstasy use to their GP in this study. An urban rather than rural research location, where there is considerable anonymity and a higher transient population, might have contributed to a greater willingness to disclose illicit drug use. Moreover, among illicit drugs, not all are equally stigmatized. Ecstasy use may be perceived as more 'acceptable' which could have contributed to greater disclosure and a more supportive response from GPs. The demographic characteristics of GPs (not determined in this study) are also likely to have played a part. For example, city- 
que tener en cuenta las características demográficas de los MC (no definidas en este estudio). Por ejemplo, los habitantes de las ciudades, de "treinta y tantos» años, tal vez incluso clientes de discotecas, tienden a manifestar menos prejuicios ante el consumo de éxtasis de sus pacientes. Las conclusiones del estudio, por tanto, son más representativas de las actitudes de los MC que ejercen en zonas urbanas deprimidas y entornos cosmopolitas.

Sería conveniente que los MC conociesen los historiales de consumo de drogas recreativas de sus pacientes. Sin embargo, dada la prevalencia relativamente baja del consumo de éxtasis (p.e., cálculos de prevalencia anual en porcentajes de población: Europa, 0,54; Estados Unidos, 0,8160), no sería relevante ni practicable que los MC preguntasen a todos los jóvenes si consumen éxtasis. Aunque lo preguntasen, muchos pacientes se resistirian a confesar el consumo de una droga ilegal. Sin embargo, los MC están en disposición de atender a los individuos con problemas relacionados con las drogas. En las zonas urbanas deprimidas, en las que hay mayor consumo de drogas ilegales, el MC debe estar al tanto del creciente consumo de fármacos de prescripción en los ambientes de discotecas y de los daños provocados por el consumo concomitante con drogas ilegales, sobre todo cuando prescribe fármacos a jóvenes.

Como todos los estudios, éste tiene serias limitaciones. Se basó en el recuerdo retrospectivo de los efectos de las drogas comunicado espontáneamente por los participantes y, por tanto, puede estar sesgado e influido por los efectos ya asumidos de las drogas. Factores como la dosis de éxtasis $^{61}$, el consumo de otras drogas ${ }^{62}$, el entorno físico ${ }^{63,64}$, el ambiente social y las expectativas ${ }^{65}$, edad ${ }^{3}$ y género ${ }^{4}$ influyen en los efectos de éxtasis y explican la diferencia entre los efectos subjetivos declarados. La muestra de consumidores de éxtasis habitantes de ciudades y de treinta y tantos años limita la generalización de las conclusiones a individuos de otros grupos de edad y de zonas no urbanas.

\section{Consecuencias}

Las conclusiones del estudio tienen importantes consecuencias en las estrategias de reducción del daño entre clientes de discotecas y destacan una serie de puntos que merecen investigaciones posteriores. Está justificado el desarrollo de mensajes saludables para informar a los consumidores de los daños potenciales de combinar éxtasis con fármacos y de la reducción de los mismos. Dicha campaña se ha lanzado y evaluado recientemente en Australia ${ }^{23}$. Hacen falta estrategias innovadoras para llegar a los usuarios que ya tienen conciencia de los riesgos potenciales. Entre los consumidores de éxtasis que legalmente toman antidepresivos por enfermedad, la evidencia de un condicionante aprendido a la hora de alterar o no la medicación antidepresiva supone un reto adicional para los educadores. La difusión de información entre consumidores de éxtasis a través de organizaciones educativas integradas por pares (p. e., KIS, Manly Drug Education and Counselling Centre, Sidney, www. kis.org.au; UNITY, Jellinek Prevention, Ámsterdam, www.unitydrugs.nl; CREW 2000, Edimburgo, www.crew2000.org.uk ) dwelling, 'thirty-something', possibly even club-going GPs, are likely to be less judgemental of their patients' ecstasy use. Study findings are, therefore, likely to be more indicative of the attitudes of GPs practicing in inner-city, cosmopolitan locations rather than elsewhere.

It makes good sense that GPs should be aware of their patients' recreational drug use histories. However, given the relatively low prevalence of ecstasy use (e.g., annual prevalence estimates in percent of population: Europe, 0.54 ; North America, 0.8160 ), it is perhaps neither relevant nor feasible that every young person presenting to a GP is asked about ecstasy use. Even if asked, patients may be reluctant to disclose their illicit drug use. GPs are, however, well placed to respond to individuals with drug-related problems. In inner-city areas where illicit drug use is more likely, GPs must be cognizant of the increasing trend in prescription drug misuse on the club scene and the associated harms of concomitant use with illicit drugs, particularly when prescribing pharmaceuticals to young people.

As with all studies, this study had several limitations. The study relied on the retrospective recall of drug effects that were spontaneously reported by participants, and may be subject to bias and the influence of learned drug effects. Factors such as ecstasy dose 61 , other drug use ${ }^{62}$, physical environment ${ }^{63,64}$, social setting and user expectancies ${ }^{65}$, age 3 and gender 4 have been found to influence the effects of ecstasy and may also account for the different subjective effects reported. The sample of city-dwelling ecstasy users in their thirties may limit the generalisability of findings to clubbers of other age groups, and those outside urban areas.

\section{Implications}

Study findings have important implications for harm reduction strategies among clubbers and highlight a number of areas for further research. The development of health messages to inform users of the potential harms of combining ecstasy with pharmaceutical drugs, and how to reduce them, is warranted. Such a campaign was recently launched and evaluated in Australia ${ }^{23}$. Innovative strategies are required to reach those users who are already aware of the potential risks. Among ecstasy users who legitimately take antidepressants for a health condition, evidence of a learned determinant to whether or not they make changes to their antidepressant regime pose additional challenges for educators. Disseminating information to ecstasy users through established peer-led education organisations (e.g. KIS, Manly Drug Education and Counselling Centre, Sydney, www.kis.org.au; UNITY, Jellinek Prevention, Amsterdam, www.unitydrugs.nl; CREW 2000, Edinburgh, www.crew2000. org.uk ) would be a way forward. A comprehensive response from GPs requires early identification and ongoing support 
supondría un gran progreso. Una respuesta completa de los $\mathrm{MC}$ exige primero una identificación y el apoyo posterior a los pacientes con síntomas de consumo de drogas ilegales, como la depresión asociada al éxtasis. Por tanto, hay que estudiar estrategias potenciales para mejorar la eficacia de la detección del consumo de éxtasis, especialmente en zonas en las que el consumo de drogas es más probable y a través de conferencias impartidas por jóvenes. Poco se sabe de los efectos del éxtasis y de su capacidad de interacción con los fármacos, y sigue habiendo un gran hueco en el conocimiento de los motivos y estrategias del consumo concomitante. Un estudio prospectivo que investigue este punto, a ser posible basándose en una amplia cohorte de consumidores de éxtasis, contribuiría a mejorar los conocimientos actuales. Atender a estos aspectos es muy importante, puesto que en muchos paises desarrollados se dejan de lado la reducción de daños y la prevención para centrar los recursos en el tratamiento coercitivo de los consumidores de drogas problemáticos dentro del sistema de justicia criminal.

\section{REFERENCIAS / REFERENCES}

1 United Nations Office on Drugs and Crime. World Drug Report 2005. Vienna: United Nations; 2005.

2 McCaughan J, Carlson R, Falck R, Siegal H. From "Candy Kids" to "Chemi-kids": a typology of young adults who attend raves in the midwestern United States. Subst Use Misuse 2005;40:1503-23.

3 Topp L, Hando J, Dillon P, Roche A, Solowij N. Ecstasy use in Australia: patterns of use and associated harm. Drug Alcohol Depend 1999;55:105-15.

4 Ter Bogt T, Engels R. 'Partying' hard: party style, motives for and effects of MDMA use at rave parties. Subst Use Misuse 2005;40:1479-502.

5 Scholey A, Parrott A, Buchanan T, Heffernan T, Ling J, Rodgers B. Increased intensity of ecstasy and polydrug usage in the more experienced recreational ecstasy/MDMA users: A WWW study. Addict Behav 2004;29:743-52.

6 Tossmann $\mathrm{P}$, Boldt $\mathrm{S}$, Tensil M. The use of drugs within the techno party scene in European Metropolitan cities. Eur Addict Res 2001;7(1):2-23.

7 Winstock A, Griffiths P, Stewart D. Drugs and the dance music scene: A survey of current drug use patterns among a sample of dance music enthusiasts in the UK. Drug Alcohol Depend 2001;64:9-17.

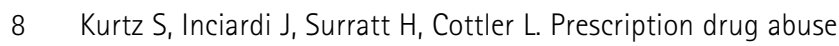
among ecstasy users in Miami. J Addict Dis 2005;24(4):1-16.

9 Black E, Dunn M, Degenhardt L, Campbell G, George J, Kinner $S$, et al. Australian trends in ecstasy and related drug markets 2007: Findings from the Ecstasy and Related Drugs Reporting System (EDRS). Australian Drug Trends Series No. 10. Sydney: National Drug and Alcohol Research Centre; University of New South Wales; 2008.

10 McCambridge J, Mitcheson L, Hunt N, Winstock A. The rise of Viagra among British illicit drug users: 5-year survey data. Drug Alcohol Rev 2006;25:111-3. of patients who present with symptoms of illicit drug use, such as ecstasy-related depression. There is, therefore, a need to explore potential strategies to improve the efficacy of screening for ecstasy use, particularly in locations where drug use is more likely and during presentations by young people. Little is known of the effects of ecstasy and its potential to interact with pharmaceutical drugs, and large gaps remain in knowledge about the motives and strategies for concomitant use. A prospective study investigating this, preferably utilising a large cohort of ecstasy users, would contribute greatly to current knowledge. Addressing these aspects is especially important as in many developed countries there is a move away from harm reduction and prevention as resources are increasingly focused on coercive treatment for problem drug users within the criminal justice system.

11 Inciardi J, Surratt H, Kurtz S, Cicero T. Mechanisms of prescription drug diversion among drug-involved club- and street-based populations. Pain Med 2007;8(2):171-83.

12 Zito J, Safer D, DosReis S, Gardner J, Madger L, Soeken K, et al. Psychotrophic practice patterns for youth: A 10-year perspective. Arch Pediatr Adol Med 2003;157:17-25.

13 Wong I, Murray M, Camilleri-Novak D, Stephens P. Increased prescribing trends of paediatric psychotrophic medications. Arch Dis Child 2004;89:1131-2.

14 Wong I, Camilleri-Novak D, Stephens P. Rise in psychotrophic drug prescribing in children in the UK - an urgent public health issue. Drug Saf 2003;26:1117-8.

15 Mant A, Rendle V, Hall W, Mitchell P, Montgomery W, McManus $P$, et al. Making new choices about antidepressants in Australia: the long view 1975-2002. Med J Aust 2004;181(7):s21-s4.

16 Wu L, Pilowsky D, Schlenger W, Galvin D. Misuse of methamphetamine and prescription stimulants among youths and young adults in the community. Drug Alcohol Depend 2007;89:195-205.

17 Substance Abuse and Mental Health Services Administration. Overview of findings from the 2003 National Survey on Drug Use and Health. Rockville, MD: Substance Abuse and Mental Health Services Administration, Office of Applied Studies; 2004.

18 International Narcotics Control Board. Report of the International Narcotics Control Board for 2006. New York: United Nations; 2007.

19 Levine S, Coupey M. Nonmedical use of prescription medications: an emerging risk behaviour among rural adolescents. J Adol Health 2009;44:407-9.

20 Kelly B, Parsons J. Prescription drug misuse among club drugusing young adults. Am J Drug Alcohol Abuse 2007;33(6):875-84.

21 Aldridge J, Measham F. Sildenafil (Viagra) is used as a recreational drug in England. Br Med J 1999;318:669.

22 Copeland J, Dillon P, Gascoigne M. Ecstasy and the concomitant use of pharmaceuticals. Addict Behav 2006;31:367-70.

23 Bleeker A, Silins E, Dillon P, Simpson M, Copeland J, Karina $H$. The feasibility of peer-led interventions to deliver health information to ecstasy and related drug users. NDARC Technical 
Report No. 299. Sydney: National Drug and Alcohol Research Centre; University of New South Wales; In press.

24 Gillman P. A review of serotonin toxicity data: Implications for the mechanisms of antidepressant drug action. Biol Psychiatry 2006;59:1046-51.

25 Silins E, Copeland J, Dillon P. A qualitative review of serotonin syndrome, ecstasy (MDMA) and the use of other serotonergic substances: hierarchy of risk. Aust N Z J Psychiatry 2007;41(8):649-55.

26 Swearingen S, Klausner J. Sildenafil use, sexual risk behaviour, and risk for sexually transmitted diseases, including HIV infection. Am J Med 2005;118:571-7.

27 Romanelli F, Smith K. Recreational use of sildenafil by HIVpositive and -negative homosexual/bisexual males. Annals Pharmacotherapy 2004;38:1024-30.

28 Deehan A, Marshall EStrang J. Tackling alcohol misuse: opportunities and obstacles in priamary care. $\mathrm{Br} J$ Gen Pract 1998;48:1779-82.

29 Roche A. Medical practitioners' involvement in drug and alcohol problems: progress and barriers. Subst Abuse 1993;14:106-16.

30 Sanson-Fisher R, Webb G, Reid A. The role of the medical practitioner as an agent for disease prevention. Better Health Commission: Looking forward to better health 1986;3:201-12.

31 Stafford J, Sindicich N, Burns L. Australian Drug Trends 2008: Findings from the Illicit Drug Reporting System (IDRS). Australian Drug Trends Series No. 19. Sydney: National Drug and Alcohol Research Centre; University of New South Wales; 2009.

32 Blum R, Beuhring T, Wunderlich M, Resnick M. Don't ask, they won't tell: the quality of adolescent health screening in five practice settings. Am J Public Health 1996;86(12):1767-72.

33 Friedman , McCullough D, Saitz R. Screening and intervention for illicit drug abuse. A national survey of primary care physicians and psychiatrists. Arch Intern Med 2001;161:248-51.

34 Maheux B, Haley N, Rivard M, Gervais A. Do physicians asses lifestyle health risks during general medical examinations? A survey of general practitioners and obstetrician-gynecologists in Quebec. JAMC 1999;160(13):1830-4.

35 Beck A, Steer R. Beck Anxiety Inventory Manual. San Antonio: Psychological Corporation 1990.

36 Beck A, Steer R, Brown G. Beck Depression Inventory Manual (2nd Edition). San Antonio: Psychological Corporation 1996.

37 Keyes K, Martin S, Hasin D. Past 12-month and lifetime comorbidity and polydrug use among young adults in the United States: Results from the National Epidemiologic Survey on Alcohol and Related Conditions. Drug Alcohol Depend 2008:97:139-49.

38 Parrott A, Milani R, Parmer R, Turner J. Recreational ecstasy/ MDMA and other drug users from the UK and Italy: psychiatric symptoms and psychobiological problems. Psychopharmacology 2001;159:77-82.

39 de Win $M$, Jager $G$, Vervaeke $H$, Schilt T, Reneman L, Booij J, et al. The Netherlands XTC Toxicity (NeXT) study: objectives and methods of study investigating causality, course and clinical relevance. Int J Methods Psychiatr Res 2005;14(4):167-85.

40 Mechan A, Esteban B, O'Shea E, Elliot J, Colado MGreen A. The pharmacology of the acute hyperthermic response that follows administration of 3,4-methylenedioxymethamphetamine (MDMA, 'ecstasy') to rats. Br J Pharmacol 2002;135:170-80.
41 Gillman P. Monoamine oxidase inhibitors, opiod analgesics and serotonin toxicity. Br J Anaesth 2005;95(4):434-41.

42 Eli Lilly and Company. Prescribing Information: Prozac, fluoxetine hydrochloride [cited 2009 June 14th]; Available from: http://pi.lilly.com/us/prozac.pdf?reqNavld=undefined

43 Gillman P. Serotonin syndrome: history and risk. Fundam Clin Pharmacol 1998;12:482-91.

44 Liechti M, Bauman C, Gamma A, Vollenweider F. Acute psychological effects of 3,4-methylenedioxymethamphetamine (MDMA, 'ecstasy') are attenuated by the serotonin uptake inhibitor citalopram. Neuropsychopharmacology 2000;22(5):513-21.

45 Vuori E, Henry J, Ojanpera I, Nieminen R, Salovainen T, Wahlsten $P$, et al. Death following ingestion of MDMA (ecstasy) and moclobemide. Addiction 2003;98:365-8.

46 Nandwani $R$, Gourlay Y. Possible interaction between sildenafil and HIV combination therapy. Lancet 1999;353:840.

47 Goldmeier D, Lamba H. Prolonged erections produced by dihydrocodeine and sildenafil. Br Med J 2002;324:1555.

48 Smith K, Romanelli F. Recreational use and misuse of phosphodiesterase 5 inhibitors. J Am Pharm Assoc 2005;45(1):63-72.

49 Alpert J. Viagra: the risks of recreational use. Am J Med 2005;118(6):569-70.

50 Vollenweider F, Gamma A, Lietchi M, Huber T. Psychological and cardiovascular effects and short-term sequelea of MDMA ('Ecstasy') in MDMA-naive healthy volunteers. Neuropsychopharamcology 1998;19:241-51.

51 Parrott A, Lasky J. Ecstasy (MDMA) effects upon mood and cognition: before, during and after a Saturday night dance. Psychopharamcology 1998;139:261-8.

52 Parrott A. Is ecstasy MDMA? A review of the proportion of ecstasy tablets containing MDMA, their dosage levels, and the changing perceptions of purity. Psychopharamcology (Berl) 2004;173:234-41.

53 Tanner-Smith E. Pharmacological content of tablets sold as 'ecstasy': Results from an online testing service. Drug Alcohol Depend 2006;83:247-54.

54 Spruit I. Monitoring synthetic drug markets, trends, and public health. Subst Use Misuse 2001;36(1):23-47.

55 Green R, Moore D. 'Kiddie drugs' and controlled pleasure: Recreational use of dexamphetamine in a social network of young Australians. Int J Drug Policy. In press.

56 Cunningham J, Sobell L, Chow V. What's in a label? The effects of substance types and labels on treatment considerations and stigma. J Stud Alcohol 1993;54:693-9.

57 Link B, Struening E, Rahav M, Phelan J, Nuttbrock L. On stigma and its consequences: evidence from a longitudinal study of men with dual diagnosis of mental illness and substance abuse. J Health Soc Behav 1997;38:177-90.

58 Kurtz S, Surratt H, Kiley M, Inciardi J. Barriers to health and social services for street-based sex workers. J Health Care Poor Underserved 2005;16:345-61.

59 Miller N, Sheppard L, Colenda C, Magen J. Why physicians are unprepared to treat patients who have alcohol- and drugrelated disorders. Acad Med 2001;76:410-8.

60 United Nations Office on Drugs and Crime. Amphetamines and ecstasy: 2008 global ATS assessment. Vienna: United Nations Office on Drugs and Crime; 2008. 
61 Zervogiannis $F$, Wiechers $E$, Bester $G$. The ' $E$ ' in rave: a profile of young ecstasy (MDMA) users. S Afr J Psychol 2003;33:162-9.

62 Gouzoulis-Mayfrank E, Daumann J. The confounding problem of polydrug use in recreational ecstasy/MDMA users: a brief overview. J Psychopharmacology 2006;20(2):188-93.

63 Parrott A. MDMA (3,4-Methylenedioxymethamphetamine) or Ecstasy: The neuropsychological implications of taking it at dances and raves. Neuropsychobiology 2004;50:329-35.
64 Parrott A. MDMA in humans: factors which affect the neuropsychobiological profiles of recreational ecstasy users, the integrative role of bioenergetic stress. J Psychopharmacology 2006;20(2):147-63.

65 McElrath K, McEvoy K. Negative experiences on ecstasy: the role of drug, set and setting. J Psychoactive Drugs 2002;34(2):199208. 